\title{
Enhancing colorimetric loop-mediated isothermal amplification speed and sensitivity with guanidine chloride
}

\author{
Yinhua Zhang', Guoping Ren'1, Jackson Buss ${ }^{1}$, Andrew J Barry', Gregory C Patton' \& Nathan A Tanner*, \\ ${ }^{1}$ New England Biolabs, Inc. 240 County Road, Ipswich, MA 01938, USA; *Author for correspondence: tanner@neb.com
}

BioTechniques 69: 179-185 (September 2020) 10.2144/btn-2020-0078

First draft submitted: 1 June 2020; Accepted for publication: 16 June 2020; Published online: 8 July 2020

\section{ABSTRACT}

Loop-mediated isothermal amplification (LAMP) is a versatile technique for detection of target DNA and RNA, enabling rapid molecular diagnostic assays with minimal equipment. The global SARS-CoV-2 pandemic has presented an urgent need for new and better diagnostic methods, with colorimetric LAMP utilized in numerous studies for SARS-CoV-2 detection. However, the sensitivity of colorimetric LAMP in early reports has been below that of the standard RT-qPCR tests, and we sought to improve performance. Here we report the use of guanidine hydrochloride and combined primer sets to increase speed and sensitivity in colorimetric LAMP, bringing this simple method up to the standards of sophisticated techniques and enabling accurate, high-throughput diagnostics.

\section{METHOD SUMMARY}

This study describes enhancing loop-mediated isothermal amplification through the addition of guanidine chloride and the use of multiple primer sets for different gene targets combined in a single reaction. We demonstrate a five- to tenfold increase in sensitivity for colorimetric SARS-CoV2 detection, further improved by use of absorbance measurement using a standard plate reader format. These alterations to the simple and rapid colorimetric loop-mediated isothermal amplification method increase performance and can be used as the basis for sensitive isothermal molecular diagnostic tests.

KEYWORDS:

high-throughput screening • isothermal amplification • LAMP • molecular diagnostics • PCR • SARS-CoV-2

Loop-mediated isothermal amplification (LAMP) was developed as a simple nucleic acid amplification technique that reliably detects target sequence at a single reaction temperature without the need for sophisticated thermal cycling equipment [1]. Advances in various detection technologies have helped establish LAMP as a useful and versatile tool for molecular diagnostics [2]. Previously we reported a reliable visual detection method based on $\mathrm{pH}$ change during LAMP, enabling simple and low-cost applications suitable for point-of-care or field diagnostics [3]. Colorimetric LAMP has been used in a range of applications; for example, a large-scale survey of Wolbachiacontaining mosquitos [4], detection of grapevine red blotch virus without DNA extraction [5] and testing urine samples for Zika virus [6]. In addition to use at the point of need, this detection method is amenable to medium- to high-throughput screening of large numbers of samples.

The recent and ongoing pandemic caused by the novel coronavirus SARS-CoV-2 has created an urgent demand for molecular diagnostics, requiring the rapid creation of accurate and sensitive tests at unprecedented scale. LAMP presents an attractive option for diagnostic testing due to its compatibility with simple colorimetric detection methods and relatively unpurified sample input; several studies have demonstrated its usage in diagnosis of SARS-CoV-2 [7,8,9; (preprints),10, 11,12,13,14 (preprints)], including its potential utility for both simple, rapid testing and higher throughput applications [11,13; preprints]. To date, these studies have demonstrated excellent specificity with LAMP, however, its sensitivity has generally been lower than that of typical RT-qPCR assays (e.g., $87.5 \%$ sensitivity as compared with a standard RT-qPCR test) [15; preprint].

Accordingly, we set out to improve the sensitivity of reverse transcription LAMP (RT-LAMP), screening new and published primer sets for SARS-CoV-2 RNA detection, and identified two sets with marked increase in sensitivity. We also screened many compounds and reaction conditions for their potential to improve LAMP speed and detection sensitivity, with the addition of guanidine hydrochloride providing a notable enhancement to both. Here we describe these studies and the performance improvement resulting from use of guanidine and the combination of primer sets together in one LAMP reaction. Using the absorbance measurement of the color change of the $\mathrm{pH}$-sensitive dye phenol red enabled more sensitive detection of low-copy amplification, reliably calling positive amplification down to ten copies of input RNA in 20 min. These modifications to standard RT-LAMP conditions will further the ability of the method to support sensitive molecular diagnostic tests, for the current COVID-19 crisis and future diagnostic needs. 


\begin{tabular}{|c|c|}
\hline Primer set & Sequence \\
\hline \multicolumn{2}{|l|}{ Gene N [15] } \\
\hline F3 & TGGCTACTACCGAAGAGCT \\
\hline B3 & TGCAGCATTGTTAGCAGGAT \\
\hline FIP & TCTGGCCCAGTTCCTAGGTAGTCCAGACGAATTCGTGGTGG \\
\hline BIP & AGACGGCATCATATGGGTTGCACGGGTGCCAATGTGATCT \\
\hline LF & GGACTGAGATCTTTCATTTTACCGT \\
\hline LB & ACTGAGGGAGCCTTGAATACA \\
\hline \multicolumn{2}{|l|}{ As1e [12] } \\
\hline F3 & CGGTGGACAAATTGTCAC \\
\hline B3 & СTTCTCTGGATTTAACACACTT \\
\hline FIP & TCAGCACACAAAGCCAAAAATTTATTTTTCTGTGCAAAGGAAATTAAGGAG \\
\hline BIP & TATTGGTGGAGCTAAACTTAAAGCCTTTTCTGTACAATCCCTTTGAGTG \\
\hline LF & TTACAAGCTTAAAGAATGTCTGAACACT \\
\hline LB & TTGAATTTAGGTGAAACATTTGTCACG \\
\hline \multicolumn{2}{|l|}{ N2 } \\
\hline F3 & ACCAGGAACTAATCAGACAAG \\
\hline B3 & GACTTGATCTTTGAAATTTGGATCT \\
\hline FIP & TTCCGAAGAACGCTGAAGCGGAACTGATTACAAACATTGGCC \\
\hline BIP & CGCATTGGCATGGAAGTCACAATTTGATGGCACCTGTGTA \\
\hline LF & GGGGGCAAATTGTGCAATTTG \\
\hline LB & CTTCGGGAACGTGGTTGACC \\
\hline \multicolumn{2}{|l|}{ E1 } \\
\hline F3 & TGAGTACGAACTTATGTACTCAT \\
\hline B3 & TTCAGATTTTTAACACGAGAGT \\
\hline FIP & ACCACGAAAGCAAGAAAAAGAAGTTCGTTTCGGAAGAGACAG \\
\hline BIP & TTGCTAGTTACACTAGCCATCCTTAGGTTTTACAAGACTCACGT \\
\hline LF & CGCTATTAACTATTAACG \\
\hline LB & GCGCTTCGATTGTGTGCGT \\
\hline \multicolumn{2}{|l|}{ АСТВ } \\
\hline F3 & AGTACCCCATCGAGCACG \\
\hline B3 & AGCCTGGATAGCAACGTACA \\
\hline FIP & GAGCCACACGCAGCTCATTGTATCACCAACTGGGACGACA \\
\hline BIP & CTGAACCCCAAGGCCAACCGGCTGGGGTGTTGAAGGTC \\
\hline LF & TGTGGTGCCAGATTTTCTCCA \\
\hline LB & CGAGAAGATGACCCAGATCATGT \\
\hline
\end{tabular}

\section{Materials \& methods}

LAMP primers targeting different regions of the SARS-CoV-2 sequence (GenBank accession number MN908947) were designed using the online software Primer Explorer V5 (https://primerexplorer.jp/e/). Primers N [15; preprint] and As1e [12; preprint] have appeared in previous preprints. We screened seven additional new primer sets and found that N2 and E1 gave best performance. Primer sequences are listed in Table 1. Oligos were synthesized at Integrated DNA Technologies with standard desalting. Synthetic SARS-CoV-2 RNA containing an equal ratio of most viral genome regions was purchased from Twist Bioscience (Twist Synthetic SARS-CoV-2 RNA Control 2 [MN908947.3] - SKU: 102024), which came at a concentration of 1 million copies/ $\mu$ l. The RNA was diluted to lower concentrations in $10 \mathrm{ng} / \mu \mathrm{l}$ Jurkat total RNA and the concentrations were estimated using RT-qPCR with primer and probe sets for CDC N1 and Charité/Berlin Gene E. RT-LAMP reactions were performed using WarmStart ${ }^{\circledR}$ Colorimetric LAMP $2 \times$ Master Mix (DNA \& RNA) (M1800) supplemented with $1 \mu \mathrm{M}$ SYTO- ${ }^{\circledR}$ double-stranded DNA binding dye (Thermo Fisher S34854) and incubated on a real-time qPCR machine (Bio-Rad CFX96) for 107 cycles with signal acquisition every $15 \mathrm{~s}$ (total incubation time $\sim 40 \mathrm{~min}$ ). The color of the finished reactions was recorded using an office flatbed scanner. Guanidine chloride solution was made with Sigma RDD001 and adjusted to $\mathrm{pH} \sim 8$ with potassium hydroxide before adding to the colorimetric LAMP reaction.

For plate reader absorbance measurement, we applied an ABI MicroAmp seal to the Bio-Rad HSP9601 microplate and incubated the reactions at $65^{\circ} \mathrm{C}$ for $20 \mathrm{~min}$ in a ThermoMixer $\mathrm{C}$ equipped with a ThermoTop heated lid and a 96-well adaptor. We then rapidly cooled 


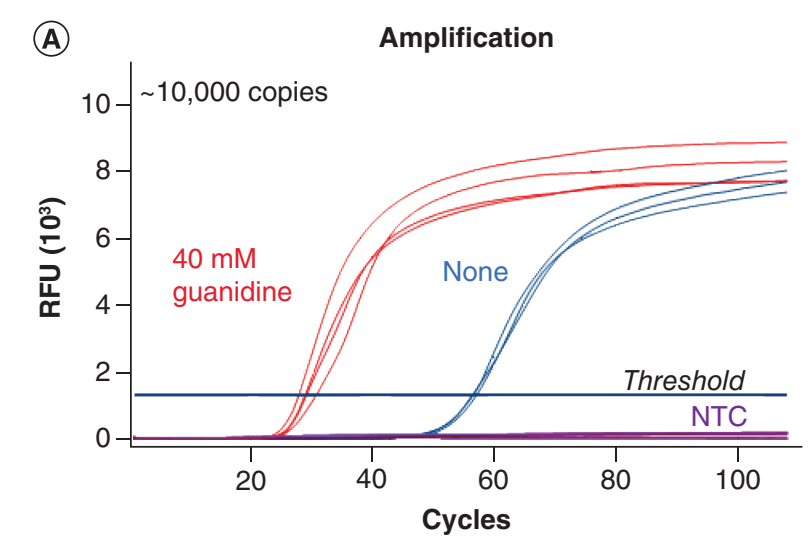

(C)

LAMP speed with or without guanidine
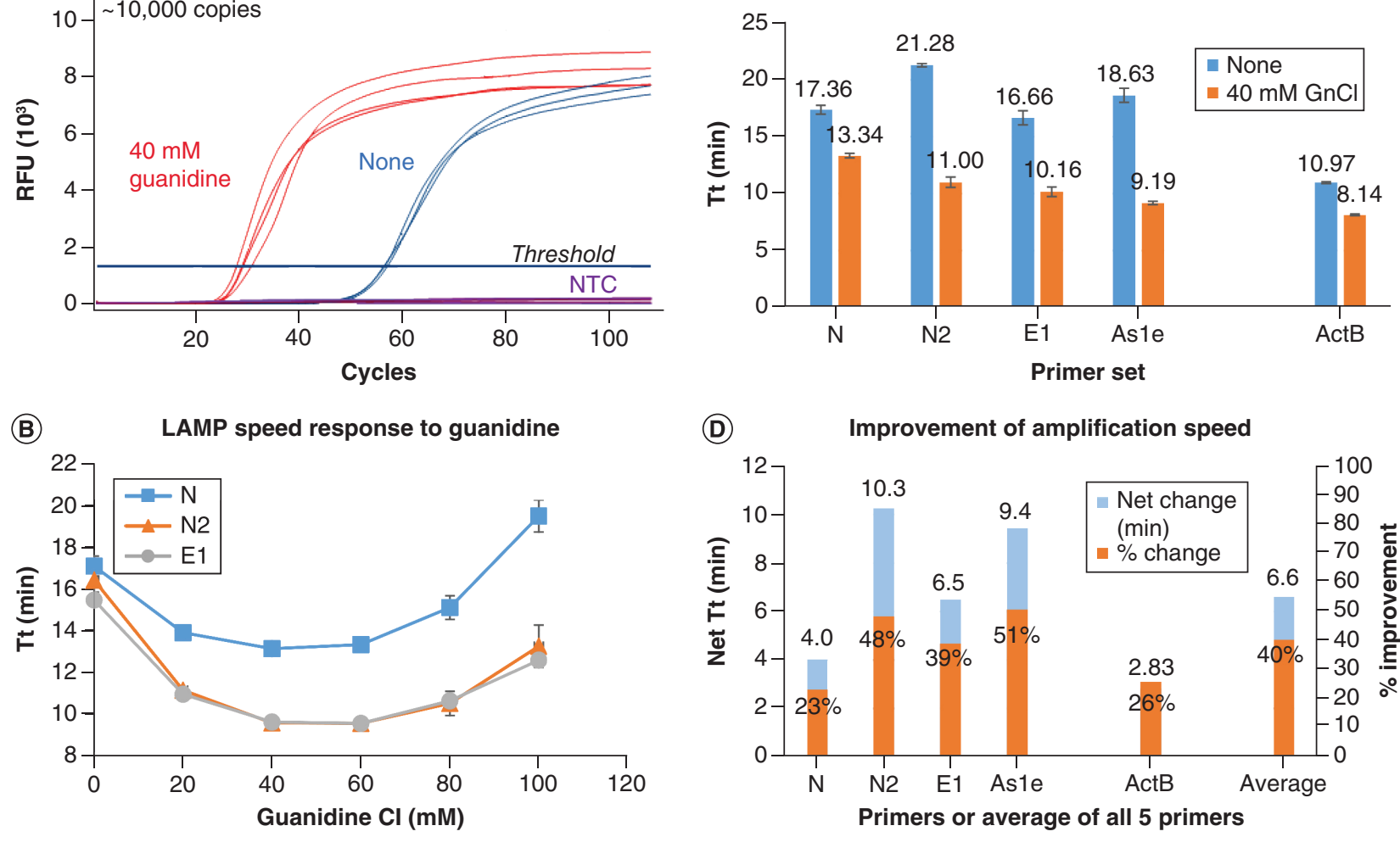

(D) Improvement of amplification speed

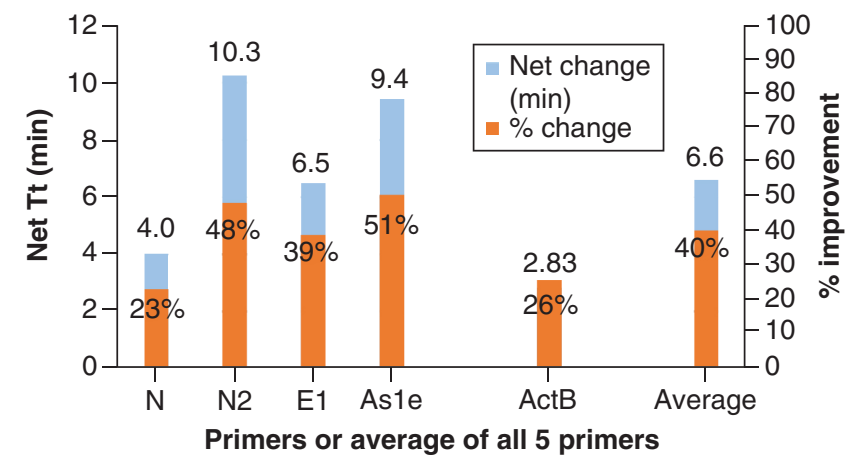

Figure 1. Increase in amplification speed by addition of guanidine chloride. (A) Real-time fluorescence curves showing increase of amplification speed by including guanidine chloride in the LAMP reaction. Speed was determined by the time to the signal crossing a threshold line (Tt) automatically set by the software. (B) Determining the optimal guanidine concentration. The speeds of three primer sets (N, N2 and E1) were plotted with averages of four repeats. (C) Amplification speeds of five primer sets, with or without guanidine chloride. There were $\sim 10,000$ copies synthetic SARS-CoV-2 RNA primers for N, N2, E1 and As1e primers. For the ACTB primer set, $1 \mathrm{ng}$ Jurkat total RNA was used in the amplification. (D) Comparing stimulation of LAMP speed by guanidine chloride. The primary $\mathrm{Y}$ axis shows the net change of $\mathrm{Tt}$ between without and with guanidine. The secondary axis shows the percentage change (net $\mathrm{Tt} / \mathrm{Tt}$ of no guanidine).

LAMP: Loop-mediated isothermal amplification; RFU: Relative fluorescence unit; Tt: Time to threshold.

the sample to reduce condensation effects and read the absorbance (SpectraMax M5; BioTek Synergy Neo2) at $432 \mathrm{~nm}$ (yellow) and $560 \mathrm{~nm}(\mathrm{red})$, corresponding to the $\mathrm{pH}$-dependent maxima of phenol red.

\section{Results \& discussion}

In order to improve the amplification in RT-LAMP reactions, we screened a range of compounds and additives commonly used to enhance PCR or other isothermal methods (e.g., DMSO, poly(ethylene glycol) compounds, betaine, single-stranded DNA-binding proteins). While most of these compounds had no, very little or negative effects on LAMP, we found that guanidine chloride showed a dramatic improvement of speed (Figure 1A). Other guanidine-containing compounds, such as guanidine thiocyanate and arginine, were also found to have a stimulatory effect, but with a narrower workable range; accordingly, we focused on guanidine hydrochloride. We determined an optimal range of guanidine chloride concentration for three primer sets used in SARS-CoV-2 RNA detection; all showed the greatest stimulation at around $40-50 \mathrm{mM}$ when used with the colorimetric LAMP master mix (Figure 1B), indicating the stimulation is likely general to colorimetric LAMP and not specific to a particular primer set.

Using this optimal concentration of guanidine, we checked the speed enhancement with four SARS-CoV-2 RNA primer sets - two for Gene N (N, N2), one for Gene E (E1) and a published set for Orf1a (As1e) - using 10,000 copies of synthetic SARS-CoV-2 RNA template and one primer set for human beta actin ( $A C T B$, often used as an extraction control assay for patient samples), with $1 \mathrm{ng}$ of total Jurkat RNA template (Figure 1C \& D). Addition of guanidine increased LAMP speed by nearly twofold for three of the primer sets: $21.3 \mathrm{~min}$ (threshold time from CFX-96 instrument) to $11 \mathrm{~min}$ for the N2 primer set; 16.7-10.2 min for the E1 primer set; and 18.3-9.2 min for the As1e primer set. The $\mathrm{N}$ primer set showed less stimulation (17.4-13.3 $\mathrm{min}$ ) as did the already very fast ACTB primer set (11-8.14 min). On average together for all five primer sets, guanidine chloride shortened the time to threshold detection by $6.6 \mathrm{~min}$, or $\sim 40 \%$. Importantly, 
(A)

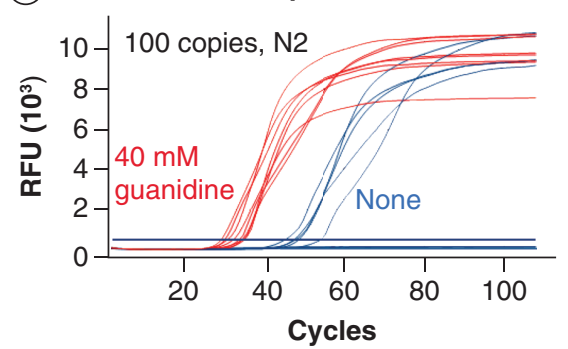

(C)

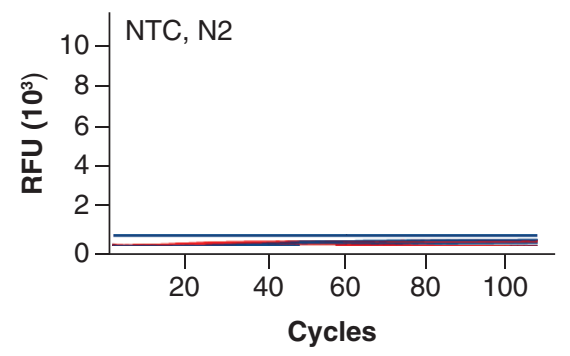

(E)

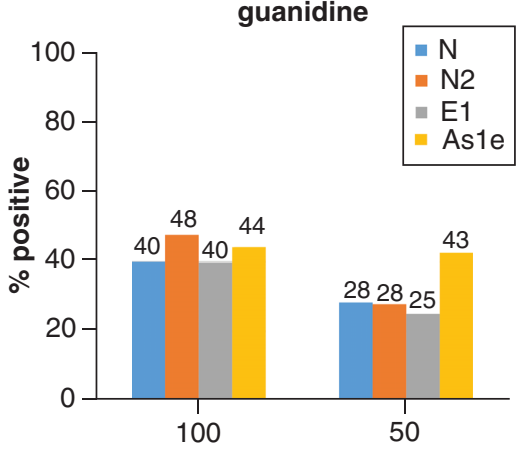

Copy number/25 ul reaction
(B)

100 copies, N2 primer set $\quad$ Positive/total

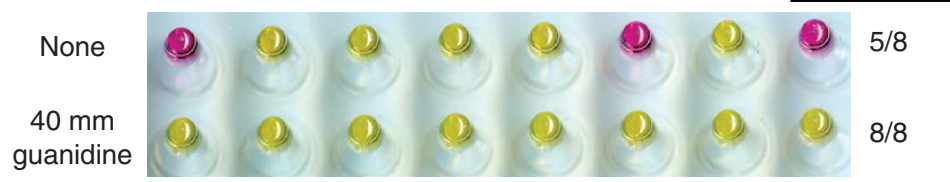

(D)

NTC, N2 primer set

Positive/total

None

$40 \mathrm{~mm}$

guanidine
(F)

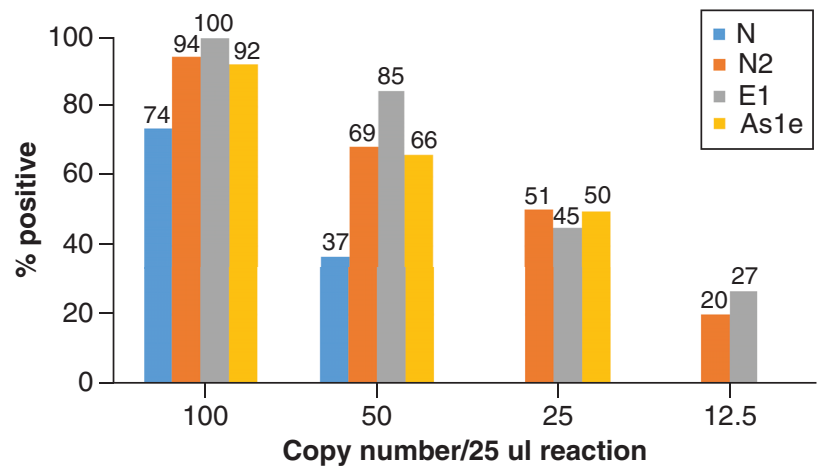

Figure 2. Enhancing detection sensitivity with guanidine chloride. (A) Real-time curve of amplifying 100 copies of SARS-CoV-2 RNA using N2 primers with or without guanidine, replicates of eight reactions each ( 1 cycle $=15 \mathrm{~s}+$ plate read). (B) End point color change scoring of positive amplification of panel A. (C) No-template control in real-time monitoring. (D) No-template control with end point color scoring of panel C. (E) Percentage amplification without guanidine with 100 and 50 copies of template RNA. (F) Percentage amplification in the presence of 40 mM guanidine with $100,50,25$ and 12.5 copies of template RNA.

this increase in detection speed did not cause any increase in no-template control amplification with any of the five primer sets (Figure 1A, Figure $2 C \& D$ ), indicating that stimulation by guanidine is specific to the interaction between primers and their intended templates.

Having observed such a significant effect on reaction speed, we next examined whether guanidine also has an effect on LAMP amplification sensitivity using lower RNA template inputs. By monitoring the reaction in real time, we found that guanidine also significantly shortened the reaction time with low template input (Figure 2A) at a similar degree to that observed with the high template input. For scoring positive amplification, we relied on end point scoring based on the color change from pink to yellow (Figure 2B); the results were concordant with real-time observations. Multiple reactions were performed for four primer sets using 100 or 50 copies of synthetic SARS-CoV-2 RNA. In all cases, guanidine significantly improved the percentage of positive detection. With 100 copies of RNA template, the detection percentage increased from just below $50 \%$ without guanidine to over $90 \%$ when it was added to reactions with the N2, E1 and As1e primer sets, with primer set $\mathrm{N}$ showing an increase from 40 to $74 \%$ (Figure 2E). With 50 copies of template, the percentage increased from $30 \%$ to around $70 \%$ (Figure 2F) for sets N2, As1e and E1. Overall, in the presence of guanidine, it is possible to successfully amplify about $50 \%$ of samples with 25 copies of input template, slightly higher than that for 100 copies in the absence guanidine. This indicates an increase in sensitivity of approximately fourfold when $40 \mathrm{mM}$ guanidine hydrochloride is included in the LAMP reaction.

Because each primer set was designed to detect different regions of the SARS-CoV-2 RNA sequence, combining two primer sets could potentially increase detection assuming no interference of the two primer sets. We tested this idea in paired combinations of 
(A)

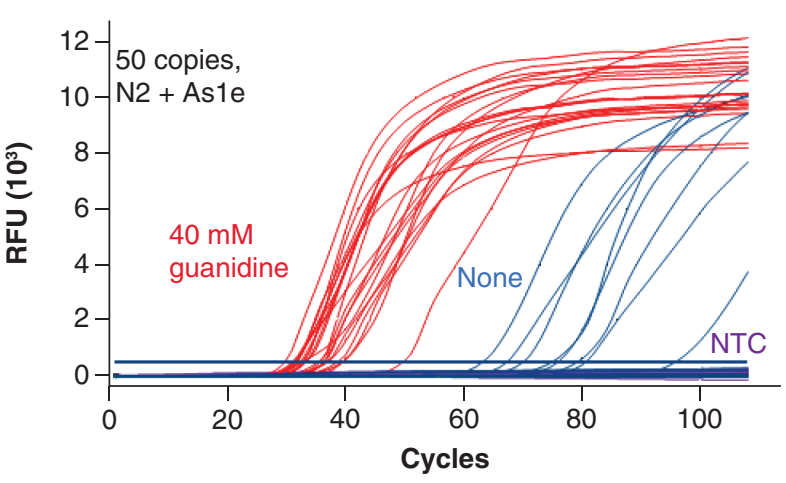

(B)
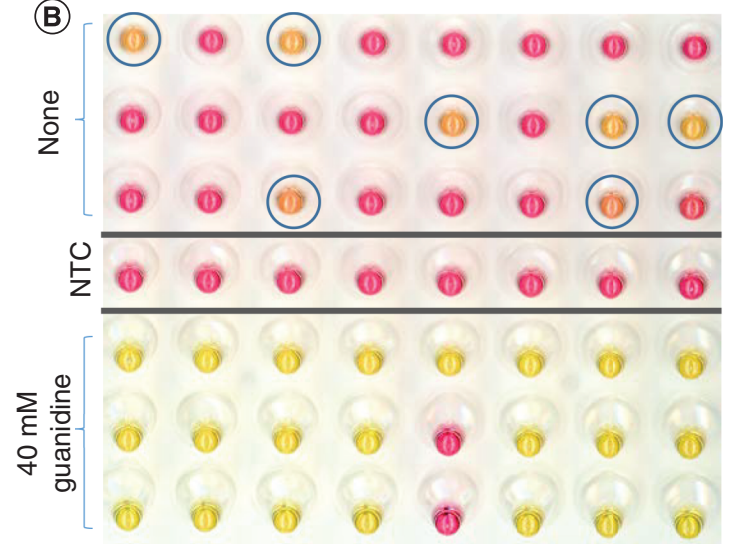

(C)

No guanidine

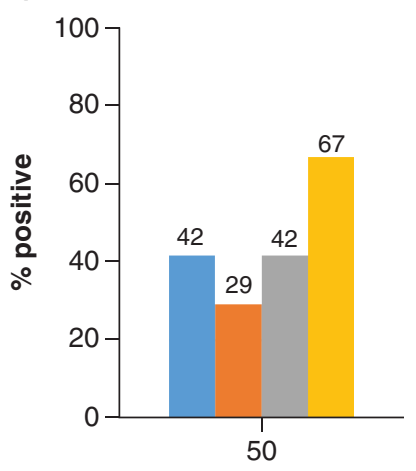

Copy number/25 ul reaction
(D)
Amplification with $40 \mathrm{mM}$ guanidine

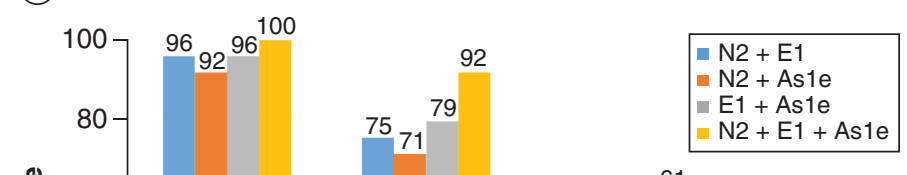

- $\mathrm{N} 2+\mathrm{E} 1$

- N2 + As1e

- 1 1 As1e

$\mathrm{N} 2+\mathrm{E} 1+\mathrm{As} 1 \mathrm{e}$

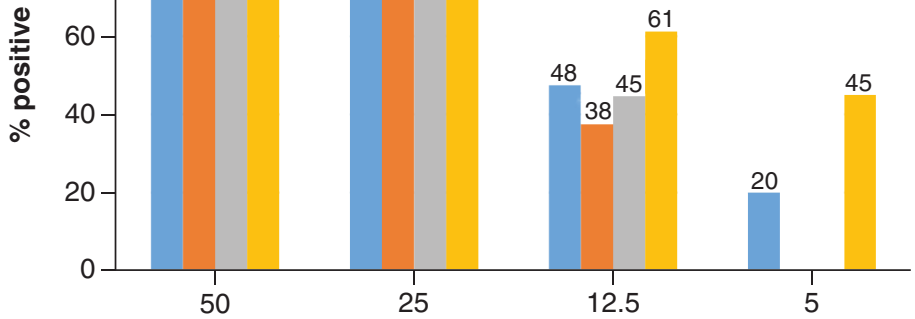

Copy number/25 ul reaction

(E) Predicted vs actual

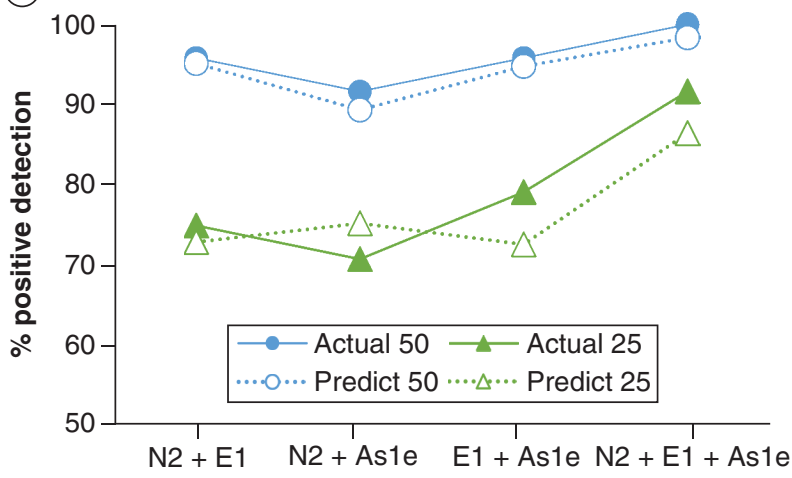

Figure 3. Guanidine enables high sensitivity detection by combining two or three primer sets. (A) Real-time curves of 24 reactions each with (red curves) or without (blue curves) guanidine, using 50 copies of SARS-CoV-2 RNA template and N2 and As1e primer sets ( 1 cycle $=15 \mathrm{~s}+$ plate read). (B) End point color change scoring for positive amplification in panel A. In the presence of guanidine, positive reactions changed to yellow completely, while in guanidine-free reactions the color change is only partially changed (circled). (C) Positive amplification rate with dual and triple primer combinations of N2, E1 and As1e primers without guanidine and with 50 copies of template. (D) Positive amplification rate with dual and triple primer combinations of N2, E1 and As1e primer sets in the presence of $40 \mathrm{mM}$ guanidine chloride with 50, 25, 12.5 and 5 copies of template. (E) Actual detection rate correlates strongly with predicted frequency.

the most sensitive LAMP primer sets (N2, E1, As1e), as well as using all three primer sets together. In these combination reactions, the concentration of each primer set was kept the same as in the reaction with only a single set, so the total primer concentrations were doubled and tripled respectively. The real-time curves of these reactions showed that, in the presence of guanidine, amplification in double and triple primer reactions started much earlier than without guanidine (Figure 3A). Almost all positive reactions started at a 


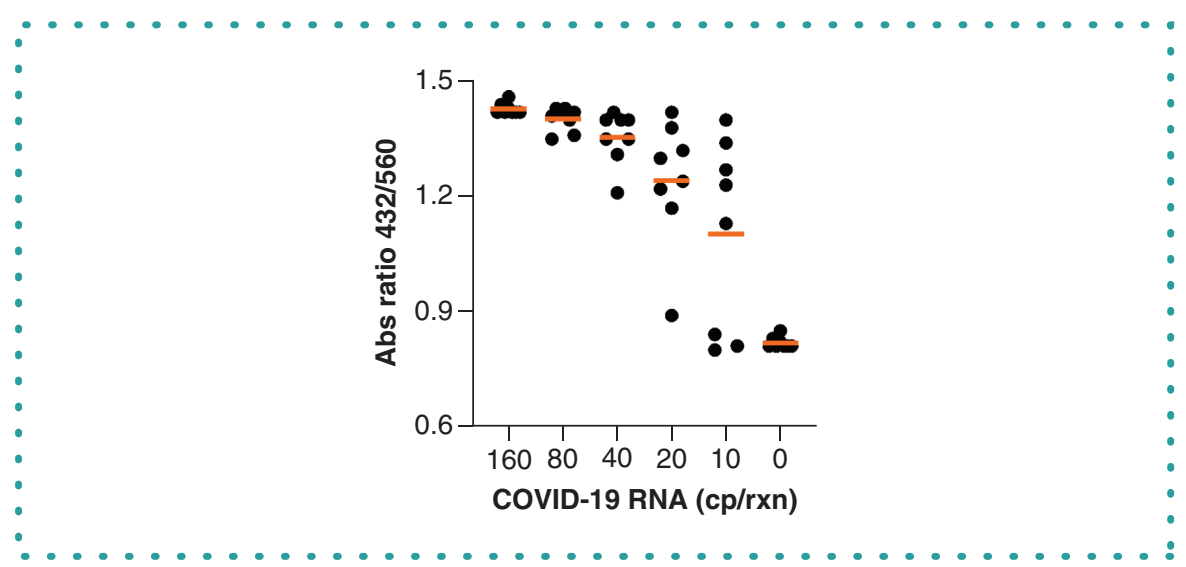

Figure 4. End point absorbance measurements of optimized colorimetric loop-mediated isothermal amplification on synthetic SARS-CoV-2 RNA. Colorimetric LAMP was performed on a synthetic SARS-CoV-2 RNA (TWIST; 160, 80, 40, 20, 10 and 0 copies/reaction) at $65^{\circ} \mathrm{C}$ for 20 min and end point absorbance at 432 and $560 \mathrm{~nm}$ was determined after a rapid cooling step. The ratio of the two absorbance readings (Abs $\left.432 / \mathrm{Abs}_{560}\right)$ is plotted against the corresponding RNA copy number $(n=8)$ and the mean for each group is displayed in orange. Results indicate a positive correlation between absorbance ratio and copy number $(\mu \pm \sigma: 160$ copies/reaction, $1.410 \pm 0.017 ; 80$ copies/reaction, $1.383 \pm 0.084 ; 40$ copies/reaction, $1.321 \pm 0.084$; 20 copies/reaction, $1.190 \pm 0.200 ; 10$ copies/reaction, $1.015 \pm 0.298 ;$ NTC, $0.679 \pm 0.008$ ), and suggest that simple, end point spectrophotometric analyses provide a rapid and sensitive approach for the detection of SARS-CoV-2 RNA by colorimetric LAMP.

LAMP: Loop-mediated isothermal amplification; NTC: No-template control.

similar early time point, while those without guanidine initiated much later, at scattered times. For the end point color change scoring, the color changed completely from pink to yellow in the presence of guanidine, while the change was only partial in reactions lacking guanidine (Figure 3B), reflecting the slower amplification.

In the absence of guanidine, the detection rate using double primers and 50 copies of RNA template only showed a slight increase, from just below $30-40 \%$ (Figures $2 \mathrm{C} \&$ Figure $3 \mathrm{C}$ ). The positive rate for the triple primer set was higher, measured at $67 \%$. When guanidine was added, all combinations showed a positive rate of over $92 \%$, with the triple primer set at $100 \%$ (Figure 3D). When even lower template amounts were tested, 25 copies were detected nearly $75 \%$ of the time with dual primer combinations and $92 \%$ with the triple primer reactions. With 12.5 copies, this rate was $45 \%$ for dual and $61 \%$ for triple primer reactions; with 5 copies, 20 and $45 \%$, respectively. These results suggest a limit of detection of $1-2$ copies/ $\mu l$ (final) in dual primer reactions and $0.5-1 \mathrm{copies} / \mu \mathrm{l}$ (final) in triple primer reactions. Importantly, in all of the dual and triple primer combination tests, there was no signal in no-template control (NTC) reactions when incubated to $40 \mathrm{~min}$ (Figure 3A \& B). Given the improvements seen, we estimate that dual primer combination in the presence of guanidine can almost double the detection frequency of SARS-CoV-2 RNA, and that the rate could be more than doubled by using all three primers. Use of all three primer sets together does show improvement in sensitivity over only the N2 and E1 combination and can be considered for maximum sensitivity, but for general use and to conserve oligonucleotide usage, the dual primer reaction represents a significant improvement over single primer reactions.

The increased detection frequency using dual or triple primers seemed to follow a simple combined detection probability. Based on the detection frequency of 50 or 25 copies with a single primer set (Figure 2F), theoretical combined detection probabilities of all combinations were calculated assuming each primer set detects its target independently and there is no interference between any combination. These predicted numbers strongly correlate with the actual detected frequency (Figure 3D) for both 50 and 25 copies (Figure $3 \mathrm{E}$ ). We also tested whether the increased detection is due to a simple primer concentration increase. When the concentration of a single primer set (N2) was doubled or tripled to match the total primer concentration of the dual and triple primer reactions, we found there was no increased detection frequency (data not shown). However, there was a noticeable increase of NTC signal. Thus the increased sensitivity is not due to simply more primer being available, but rather due to LAMP reactions of multiple primer sets occurring independently at the same time, and effectively increasing the available number of templates for the same target.

Next we sought to improve detection sensitivity and evaluate high-throughput compatibility by quantitatively detecting the colorimetric change of our reactions using spectrophotometric measurements. We combined the optimal guanidine hydrochloride concentration $(40 \mathrm{mM})$ with the dual primer sets ( $\mathrm{N} 2$ and E1) described above and performed colorimetric LAMP reactions with a twofold dilution series of synthetic SARS-CoV-2 RNA. Using the ratio of absorbance measurements of the two phenol red peaks (yellow Abs ${ }_{432} /$ pink Abs $_{560}$ ) as the primary metric, we observed a positive correlation with copy number (Figure 4). Applying a threshold of 0.704 , equivalent to the $99.7 \%$ confidence interval ( $\left.\mu_{\mathrm{NTC}}+3 \sigma_{\mathrm{NTC}}\right)$ of NTC $(n=8)$, we determined that $100 \%$ of all samples containing $20-160$ copies per reaction tested positive. Notably, $62.5 \%$ of samples containing only ten copies per reaction also tested positive, illustrating a marked improvement in sensitivity. These reactions were incubated for only $20 \mathrm{~min}$, with the more sensitive instrumented measurement enabling positives to be called much earlier than detection simply by eye. 
(A)

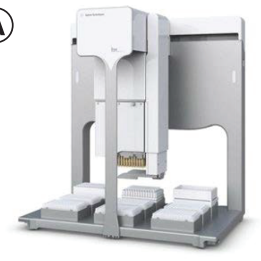

96-384 transfer tube-plate

- $3 \mu$ l of RNA template - $4 \times 96$ well tube rack - $1 \times 384$ well plate
(B)
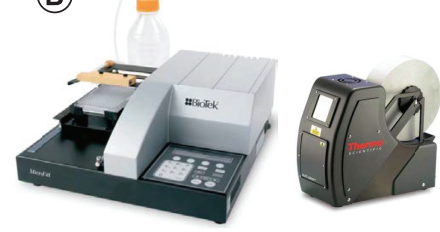

Bulk dispense of LAMP Mastermix

E.g. $20 \mu$ reaction mixture

- $4 \mu \mathrm{L} 5 \times$ primer mix with $\mathrm{GnCl}$

- $10 \mu \mathrm{L}$ WarmStart colorimetric lamp 2X master mix (M1800)

- $3 \mu \mathrm{L}$ of DNAse, RNAase free $\mathrm{H} 2 \mathrm{O}$
(C)
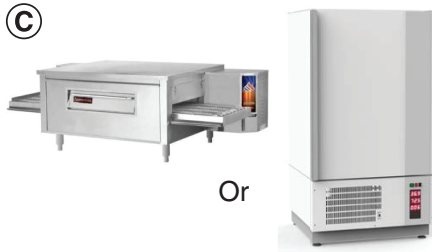

Incubation

(a) $65^{\circ} \mathrm{C}$
(D)

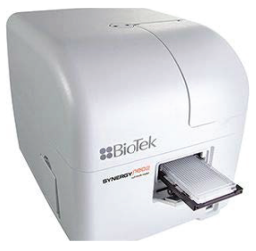

Detection

absorbance reader

- $65^{\circ} \mathrm{C}$ for $20-40$ minutes

- 384 well plate

- Potential to share oven

across workflow streams

Figure 5. Example of an automated workflow supporting high-throughput end point detection of colorimetric loop-mediated isothermal amplification. (A) 96-channel liquid handler (Agilent Bravo; Agilent, CA, USA; P/N G5563A) for transfer of extracted nucleic acids into 384-well absorbance plate. (B) Bulk dispensing of LAMP Mastermix into 384-well plates (BioTek Ufill, AF1000A) and plate sealing (ALPS AB-3000 plate sealer; ThermoFisher, MA, USA). (C) Isothermal incubation of sealed plates using a custom conveyor oven (Intek, MO, USA), or automated random-access incubator (STR240, Liconic, Mauren, Liechtenstein). (D) End point detection using absorbance plate reader (Synergy Neo2, BioTek, VT, USA).

We present here a significant increase in the speed and sensitivity of RT-LAMP reactions by the addition of guanidine hydrochloride. This effect is further enhanced by combining two or three LAMP primer sets, enabled by guanidine and resulting in additional improvement in sensitivity without any increase in nonspecific amplification. These enhanced reactions also make it possible to utilize shorter detection times and permit better discrimination of color change with the colorimetric LAMP detection based on pH-sensitive dyes.

The mechanism of action of guanidine hydrochloride in RT-LAMP is yet to be elucidated. Based on its consistent enhancement of reactions with different primer sets, however, we speculate that it enhances base pairing between primers and their target sequences. Consistent with this interpretation, guanidine addition also significantly shortens reaction times for helicase-dependent amplification (data not shown). The enhancement is likely not a result of modulation of enzyme activity, because additional enzyme, either reverse transcriptase or Bst 2.0 DNA polymerase, did not have such an effect. While the primary focus here is RT-LAMP reactions, the guanidine enhancement is equally effective with LAMP using DNA inputs (not shown).

Measurement with an absorbance plate reader also demonstrated a notable increase in reaction sensitivity, clearly calling positives that would have been indeterminate at best by visual detection. While visual readout is well suited to simple field and point-of-care applications, end point reading of plates by absorbance provides a quantitative data record that is more amenable to high-throughput settings. Plates (96 or 384 well) could be incubated in simple heating chambers and end point absorbance used for analysis; if paired with liquid handling plate filling, a large number of samples could be processed with the simple 20- to 40 -min heating at $65^{\circ} \mathrm{C}$ and end point plate read. One example of this workflow is shown in Figure 5, with automated liquid handling and nucleic acid extraction for plate setup, followed by heating in an oven and measurement of absorbance to determine the presence or absence of target. An Emergency Use Authorization was recently granted to Color Genomics for a SARS-CoV-2 test much as laid out here, proving its potential as a highthroughput workflow. Taken together, the improvements to LAMP presented here describe a significant increase in sensitivity for this powerful isothermal method to be more fully utilized for molecular diagnostics.

\section{Future perspective}

The SARS-CoV-2 pandemic has highlighted a need for a significant expansion in our ability to conduct diagnostic testing in order to track the spread of public health threats rapidly and accurately. Clinical labs and RT-qPCR tests have served, and will continue to serve, as the core testing capability; however, the ability to test at the point of care, in the workplace or even at home would dramatically enhance our ability to identify infected people. Isothermal amplification methods have already shown promise for these applications, and with enhanced performance and simplifying both high-throughput and fieldable testing, methods like LAMP can lead a shift in how we conduct molecular diagnostic surveillance.

\section{Author contributions}

Y Zhang, G Ren and J Buss conducted experiments and collected and analyzed data. Y Zhang, G Ren, J Buss, A Barry, G Patton and N Tanner conceived the study and wrote the manuscript. 


\section{Financial \& competing interests disclosure}

The authors are employees of New England Biolabs, manufacturer of reagents described in the paper. The formulation and primers described here are the basis of a research-use-only COVID LAMP test product offered by NEB. The authors have no other relevant affiliations or financial involvement with any organization or entity with a financial interest in or financial conflict with the subject matter or materials discussed in the manuscript apart from those disclosed.

No writing assistance was utilized in the production of this manuscript.

\section{Open access}

This work is licensed under the Attribution-NonCommercial-NoDerivatives 4.0 Unported License. To view a copy of this license, visit http://creativecommons.org/licenses/by-nc-nd/4.0/

\section{References}

1. Notomi T, Okayama H, Masubuchi $\mathrm{H}$ et al. Loop-mediated isothermal amplification of DNA. Nucleic Acids Res. 28(12), E63 (2000).

2. Zhang X, Lowe SB, Gooding JJ. Brief review of monitoring methods for loop-mediated isothermal amplification (LAMP). Biosens. Bioelectron. 61, 491-499 (2014).

3. Tanner NA, Zhang Y, Evans TC Jr. Visual detection of isothermal nucleic acid amplification using pH-sensitive dyes. BioTechniques 58(2), 59-68 (2015).

4. Goncalves DDS, Hooker DJ, Dong Y et al. Detecting wMel Wolbachia in field-collected Aedes aegypti mosquitoes using loop-mediated isothermal amplification (LAMP). Parasit. Vectors 12(1), 404 (2019).

5. Romero Romero JL, Carver GD, Arce Johnson P, Perry KL, Thompson JR. A rapid, sensitive and inexpensive method for detection of grapevine red blotch virus without tissue extraction using loop-mediated isothermal amplification. Arch. Virol. 164(5), 1453-1457 (2019).

6. Calvert AE, Biggerstaff BJ, Tanner NA, Lauterbach M, Lanciotti RS. Rapid colorimetric detection of Zika virus from serum and urine specimens by reverse transcription loop-mediated isothermal amplification (RT-LAMP). PLoS ONE 12(9), e0185340 (2017).

7. Baek YH, Um J, Antigua KJC et al. Development of a reverse transcription-loop-mediated isothermal amplification as a rapid early-detection method for novel SARS-CoV-2. Emerg. Microbes Infect. doi:10.1080/22221751.2020.1756698 1-31 (2020) (Epub ahead of print).

8. Butler DJ, Mozsary C, Meydan C et al. Shotgun transcriptome and isothermal profiling of SARS-CoV-2 infection reveals unique host responses, viral diversification, and drug interactions. bioRxiv doi:10.1101/2020.04.20.048066 2020.2004.2020.048066 (2020) (Epub ahead of print).

9. Butt AM, Siddique S, An X, Tong Y. Development of a dual-gene loop-mediated isothermal amplification (LAMP) detection assay for SARS-CoV-2: a preliminary study. 2020) (Epub ahead of print).

10. Huang WE, Lim B, Hsu CC et al. RT-LAMP for rapid diagnosis of coronavirus SARS-CoV-2. Microb Biotechnol doi:10.1111/1751-7915.13586 (2020) (Epub ahead of print).

11. Lalli MA, Chen X, Langmade SJ et al. Rapid and extraction-free detection of SARS-CoV-2 from saliva with colorimetric LAMP. 2020) (Epub ahead of print).

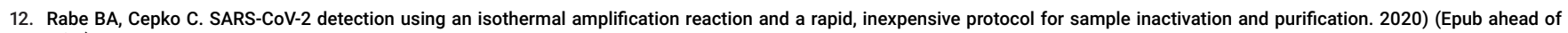
print).

13. Thi VLD, Herbst K, Boerner K et al. Screening for SARS-CoV-2 infections with colorimetric RT-LAMP and LAMP sequencing. 2020) (Epub ahead of print).

14. Anahtar MN, McGrath GE, Rabe BA et al. Clinical assessment and validation of a rapidand sensitive SARS-CoV-2 test using reverse-transcription loop-mediated isothermalamplification. medRxivdoi:2020.2005.2012.20095638 (2020).

15. Zhang Y, Odiwuor N, Xiong J, Sun L, Wei H, Tanner NA. Rapid molecular detection of SARS-CoV-2 (CoVID-19) virus RNA using colorimetric LAMP. 2020) (Epub ahead of print). 